\title{
A texture evolution model in cubic-orthotropic polycrystalline system
}

\author{
Brent L. Adams \\ b_I_adams@byu.edu \\ H. Garmestani \\ D. S. Li
}

Follow this and additional works at: https://scholarsarchive.byu.edu/facpub

Part of the Mechanical Engineering Commons

\section{Original Publication Citation}

International Journal of Plasticity 21 (25) 1591-1617

\section{BYU ScholarsArchive Citation}

Adams, Brent L.; Garmestani, H.; and Li, D. S., "A texture evolution model in cubic-orthotropic polycrystalline system" (2003). Faculty Publications. 475.

https://scholarsarchive.byu.edu/facpub/475 


\title{
A texture evolution model in cubic-orthotropic polycrystalline system
}

\author{
D.S. Li a, H. Garmestani a, ${ }^{,}$, B.L. Adams b \\ a School of Materials Science and Engineering, Georgia Institute of Technology, \\ 771 Ferst Drive, N.W. Rm 175, Atlanta, GA 30332, USA \\ b Department of Mechanical Engineering, Brigham Young University, Provo, UT 84602, USA \\ Received in final revised form 28 September 2003 \\ Available online 7 January 2005 \\ 2004 Elsevier Ltd. All rights reserved. \\ Keywords: A. Microstructure; A. Thermomechanical processes; \\ B. Polycrystalline material; B. Processing path; B. Streamline \\ 2004 Elsevier Ltd. All rights reserved. \\ doi:10.1016/j.ijplas.2004.11.009 \\ * Corresponding author. Tel.: +1 4043854495; fax: +1 4048949140. \\ E-mail address: hamid.garmestani@mse.gatech.edu (H. Garmestani). \\ www.elsevier.com/locate/ijplas \\ International Journal of Plasticity 21 (2005) 1591-1617
}

\begin{abstract}
A new methodology based on a conservation principle in the orientation space is developed to simulate the texture evolution in a cubic-orthotropic polycrystalline system. A least squares error method was used to improve the accuracy of the simulation results obtained from the texture evolution function. The model is applied to uniaxial tension, compression and rolling for a large deformation of more than $50 \%$ using a single evolution parameter. The validity and application range of this new model are discussed by simulating and predicting texture evolution during different loading conditions. The new methodology provides a family of texture evolution paths and streamlines which empowers the materials designer to optimize the desired microstructure.
\end{abstract}

\section{Introduction}

A major goal for materials design is the selection and optimization of microstructures for a specified set of properties and mechanical constraints. To achieve this goal, it is necessary to choose the right and most appropriate processing path. The solution may not be unique and the optimum path may depend on a number of parameters including cost and reliability. Traditional design methodologies attempt to relate properties to off-the-shelf materials while the range of microstructures (texture, grain size, deformation history, etc.) for each material of choice is usually ignored. Such variation in the microstructure of a specific material provides a large range of properties that may meet specific design constraints not readily available in the conventional offthe-shelf materials design.

To make a direct linkage to microstructures, Adams et al. $(2001,2004)$ proposed a novel methodology, known as microstructure sensitive design (MSD), which shifted the current paradigm for designer materials. The new methodology, as was introduced 
originally, is concentrated on texture (orientation distribution function) in polycrystalline materials as a basic variable for design. The other microstructure attributes such as grain size, grain boundary distribution, morphology, second phase particles and precipitates may be included in the framework using two and higher order statistical distribution functions (Garmestani and Lin, 2000, 2001; Lin et al., 1998, 2000; Jefferson et al., 2004). The linkage to property enclosure may require the correct form of the constitutive relations based on better understanding of the physics and the underlying deformation mechanisms. The representation of microstructure in the form of texture may assume the most basic form and by itself can cover anywhere from a single crystal (as a limiting form of the distribution function) to bi-crystals and random polycrystals. Appreciable anisotropic properties are usually possessed by single crystals which are expensive to obtain. Polycrystalline materials are usually less expensive to produce and more readily available. The incentive to cut the cost by utilizing the state of anisotropy to maximize the potential application of polycrystalline material prompts material scientists to seek the means to achieve the optimized texture by thermomechanical processing. In homogeneous polycrystalline materials texture determines the anisotropy in mechanical, thermal, magnetic and electrical properties. MSD in its present form is an approach to balance the requirements of several different properties by optimizing texture (Adams et al., 2001, 2004; Kalidindi et al., 2004). To achieve this goal, a quantitative description of material microstructure as a set of texture coefficients, which is associated with properties, is introduced as a variable in design.

Texture is commonly represented as a Fourier series of generalized spherical harmonics weighted by appropriate texture coefficients (Bunge, 1965). Using the set of texture coefficients as a descriptor, microstructures can be represented as points in a multidimensional space with coordinates as texture coefficients (Adams et al., 2001, 2004). The dimension depends on the number of the texture coefficients used. Each point in this Fourier space stands for a unique texture, associated with corresponding properties. Texture coefficients are important in determining the properties of polycrystalline materials. The properties of polycrystalline materials can be represented as a summation of the product of property coefficients and spherical harmonics:

$$
\bar{e}=\sum_{l=0}^{\infty} \sum_{m=0}^{M(l)} \sum_{n=0}^{N(l)} \bar{e}_{l}^{\mu v} \dot{\bar{T}}_{l}^{m n}(g) .
$$

While the property coefficients of a single crystal, $e_{l}^{\mu \lambda}$, are constants, the property coefficients of polycrystalline material, $\bar{e}_{l}^{\mu v}$, are dependent directly on the texture coefficients, $F_{l}^{\lambda v}$

$$
\bar{e}_{l}^{\mu v}=\frac{1}{2 l+1} \sum_{\lambda=1}^{M(l)} e_{l}^{\mu \lambda} F_{l}^{\lambda v}, \quad 0 \leqslant l \leqslant r,
$$

where $r$ is the limit of order which depends on the crystal symmetry, sample symmetry and the property $e$. It is very important to have a full mathematical representation of texture evolution during thermomechanical processing. In this paper, texture evolution is defined as a continuous line in the texture hull to represent the texture evolution. Texture is conventionally measured using X-ray diffraction as a set of pole figures. The complete representation of texture requires a number of pole figures depending on the crystal group and sample symmetry. For cubic-orthotropic case, three pole figures are usually needed to fully characterize texture. Using ODF plots has some 
other limitations. They distort the distribution geometrically in Euler space. Random distribution looks like textured in the ODF plots. Also there is a multiplicity in ODF plots, especially for high symmetry materials. Although texture can be represented in a number of ways, the spectral representation can introduce a new geometrical representation in the texture hull such that every point represents one specific microstructure (texture).

Modeling texture evolution is an important component of MSD. To modify the properties through thermomechanical deformation requires correct representation of the evolution of microstructures. A conservation principle in the orientation space proposed by Clement and Coulomb $(1979,1982)$ was used in this work to model the texture evolution. This principle refers to an infinitesimal volume element in the orientation (Euler) space during processing. Based on Clement's formalism and the continuity equation, Bunge and Esling (1984) studied the flow field of single orientations of face centered cubic (fcc) metals using a crystal plasticity formulation based on slip activity on the $\left\{\begin{array}{lll}1 & 1 & 1\end{array}\right\}<1 \quad 1 \quad 0>$ slip system. In a later work (Klein and Bunge, 1991), a numerical integration methodology was used to obtain a relationship between the texture coefficients and the deformation step. In a recent study by Li and Garmestani (2003a,b) an alternate approach using polycrystalline texture representation rather than single crystal orientation description was used to describe texture evolution. This approach established a linear relationship between the rate of change of the texture coefficients and the texture coefficients. Further progress is made in the present study for a direct relationship between texture coefficients and deformation parameter. A processing path function is proposed to describe the evolution of texture coefficients in the form of processing parameters and initial texture coefficients. To examine the accuracy and range of applicability for this approach, a modified Taylor model proposed by Kalidindi et al. (1992a, 1992b) was used for comparison. In Taylor model, it is assumed that all the individual grains in polycrystalline materials undergo the same deformation gradient as the macroscopic one. This simplification satisfies the local compatibility, but often violates equilibrium. Although the Taylor model ignores many complexities embedding in mechanical deformation, it provides a fairly accurate approximate solution for the texture evolution of single phase, highly symmetric lattice structures such as fcc polycrystals during large plastic deformation (Kalidindi et al., 1992a, 1992b; Garmestani et al., 2002).

\section{Evolution of texture coefficient during plastic deformation}

Using the conservation principle in the orientation space, a set of relationships for the evolution of texture coefficients can be derived. A formulation is presented here for the streamlines as an analytical form among the texture coefficients for any specific thermomechanical process. In the formulation presented here, $\eta$ is used as an appropriate metric of the process. For example, in the case of uniaxial tension, $\eta$ represents the drawing strain and in the case of compression, $\eta$ represents the compression ratio.

Texture descriptor used in this study is a set of $F_{l}^{m n}(\eta)$ coefficients that changes as a function of $\eta$. If $f(g, \eta)$ is used to represent texture as a function of orientation $\mathrm{g}$ and processing parameter $\eta$, texture at any $\eta$ can be expressed as a series of generalized spherical harmonic functions in which $F_{l}^{m n} \mathrm{~s}$ are the weights (coefficients) of these harmonics, as shown in the following equation: 


$$
f(g, \eta)=\sum_{l=0}^{\infty} \sum_{m=0}^{M(l)} \sum_{n=0}^{N(l)} F_{l}^{m n}(\eta) \dot{\bar{T}}_{l}^{m n}(g) .
$$

Here $\dot{\overparen{T}}_{l}^{m n}(g)$ is the symmetric generalized spherical harmonics for the corresponding sample and crystal symmetry. For a single crystal orientation distribution in which all the crystals are oriented along $g_{i}$, texture coefficients $F_{l}^{m n}$ are calculated directly from the spherical harmonics:

$$
F_{l}^{m n}=(2 l+1) \dot{T}_{l}^{m n}\left(g_{i}\right) \text {. }
$$

Texture measured from X-ray diffraction or electron backscatter diffraction (EBSD) gives the volume fraction of material with orientation $g_{i}$ (Garmestani, 1998; Garmestani et al., 1999). $F_{l}^{m n}$ can then be calculated from $f(g)$ by linear combination of the texture coefficients of single crystal orientations:

$$
F_{l}^{m n}=(2 l+1) \sum_{i} f\left(g_{i}\right) \dot{\vec{T}}_{l}^{m n}\left(g_{i}\right) .
$$

In Clement's work (1982), the texture evolution is regarded as a fluid flow in orientation space. Three Eulerian angles compose the orthogonal coordinates of this space. For any point represented by $g$, the density is $\frac{1}{8 \pi^{2}} f(g, \eta) \sin \phi$ and the flow rate is $R(g)$. According to the conservation principle in the orientation space, the sum of the increase of the quantity of matter in an element of volume $\mathrm{d} v$ and the material moving across the surface $S$ should be zero. The continuity equation can then be represented as:

$$
\oint_{S} f(g, \eta)\left(\frac{1}{8 \pi^{2}}\right) \sin \phi R \cdot \hat{n} \mathrm{~d} \sigma+\frac{\partial}{\partial t} \iint_{V} \int f(g, \eta)\left(\frac{1}{8 \pi^{2}}\right) \sin \phi \mathrm{d} v=0 .
$$

Thus,

$$
\frac{\partial f(g, \eta)}{\partial t}+\frac{1}{\sin \phi} \operatorname{div}[f(g, \eta) \sin \phi R(g)]=0 .
$$

For an infinitesimal volume element in the orientation space, $\mathrm{d} v=\mathrm{d} \varphi_{1} \mathrm{~d} \varphi \mathrm{d} \varphi_{2}$, the first term in Eq. (7) is the increase of the quantity of matter per unit time and the second term describes the quantity of matter moving out of the infinitesimal volume element. By simplifying Eq. (7), the continuity equation for the conservation of quantity of matter in a volume element in the Euler space is:

$$
\frac{\partial f(g, \eta)}{\partial t}+\operatorname{div}[f(g, \eta) R(g)]+\operatorname{ctg} \phi f(g, \eta) R(g)=0 .
$$


Using the expression for texture in Eq. (1), Eq. (8) is expanded in a series of spherical harmonics:

$$
\begin{aligned}
& \sum_{l m n} \frac{\mathrm{d} F_{l}^{m n}(\eta)}{\mathrm{d} \eta} \dot{T}_{l}^{m n}(g)+\sum_{\lambda \sigma \rho} F_{\lambda}^{\sigma \rho}(\eta)\left(\operatorname{div}\left(\dot{T}_{\lambda}^{\sigma \rho}(g) R(g)\right)+\operatorname{ctg} \phi \dot{T}_{\lambda}^{\sigma \rho}(g) R(g)\right) \\
& \quad=0 .
\end{aligned}
$$

The second summation can be further expanded into a series of generalized spherical harmonics:

$$
\operatorname{div}\left(\dot{\ddot{T}}_{\lambda}^{\sigma \rho}(g) R(g)\right)+\operatorname{ctg} \phi \dot{\bar{T}}_{\lambda}^{\sigma \rho}(g) R(g)=-\sum_{l m n} A_{l \lambda}^{m n \sigma \rho} \dot{T}_{l}^{m n}(g) .
$$

Here $A_{\lambda l}^{\sigma \rho m n}$ is introduced as the coefficients of the spherical harmonics. Substituting this back into Eq. (9), a linear relationship between the texture coefficients and their rate of change is derived:

$$
\frac{\mathrm{d} F_{l}^{m n}(\eta)}{\mathrm{d} \eta}=\sum_{\lambda \sigma \rho} A_{l \lambda}^{m n \sigma \rho} F_{\lambda}^{\sigma \rho}(\eta) .
$$

This linear relationship was used by Bunge and Esling (1984) and Klein and Bunge (1991) to predict the texture evolution in the orientation space. In this present work, a texture evolution function obtained by the integration of Eq. (11) was used to describe the evolution of the texture coefficients with the deformation parameter:

$$
F(\eta)=\mathrm{e}^{A \eta} F\left(\eta_{0}=0\right) \text {. }
$$

The coefficients represented by the sixth rank tensor A, can be rearranged as the elements of a matrix and will be called "texture evolution matrix" in this work. In this study, Eq. (12) was used to simulate the texture evolution of fcc materials with random texture to generate a process path. If the number of useful texture coefficients $F_{l}^{m n}$ is limited to $N$, then texture data at $N+1$ different strains will be needed to obtain a solution for texture evolution coefficients $A_{\lambda l}^{\sigma \rho m n}$. It will be shown that redefining the sixth rank tensor coefficients to an $N \times N$ matrix will substantially simplify the numerical scheme. The final analytical form for the texture evolution as a function of $A$ will represent the texture evolution.

The introduction of a microstructure parameter " $A$ " can be used as a methodology to predict microstructure evolution in a large set of polycrystalline materials. The sixth order tensor " $A$ " as derived in Eq. (11) (continuity relation in the Euler space) is the underlying parameter in this basic and simple law. The sixth order tensor maybe a function of many different other features of the microstructure.

$$
A_{l \lambda}^{m n \sigma \rho}=A_{l \lambda}^{m n \sigma \rho}\left(s_{1}, s_{2}, s_{3}, \ldots\right),
$$

where $s_{i}$ may represent a number of microstructure features such as grain size and distribution, precipitates, dislocation density, second phase particles which can affect the evolution. A Taylor type crystal plasticity model is used in this paper to calculate the sixth order " $A$ ". The application of Taylor may introduce a limitation in the range of applications but within the limits of Taylor, many of these microstructural parameters can be incorporated. As a first exercise, this paper concentrates on Taylor and produces enough results which will show that such continuity relations and the consequent streamlines (derived in this paper) are valid for a large range of deformation processes. The incorporation of the other alternative (crystal plasticity homogenization) methodologies can be easily incorporated at a later time. 
Texture is defined as "preferred orientation distribution" and is a macroscopic and average representation of the microstructure. A formulation based on texture does not take into account grain structure and grain boundary character and also the grain to grain interaction. Texture as an average representation is only a one point distribution function. Higher order statistics can incorporate the additional details of the microstructure (Garmestani et al., 2000, 2001; Lin et al., 1998, 2000; Jefferson et al., 2004; Adams et al., 1989; Torquato and Stell, 1985) and can be used for the evolution of the microstructure. It is clear that the evolution of texture is a function of the details and physics of the microstructure and the underlying deformation mechanism. At a first glance it seems that such details are neglected in the formulation presented in the paper. All these details are however embedded in the sixth order tensor ' $A$ ". It sounds very optimistic to expect that such a parameter can incorporate all these effects but the main goal of this paper is to investigate whether such a claim is valid and to what degree. It will be shown that the evolution can be taken care of using conservation principle for more than $50 \%$ deformation.

\section{Simulation result of texture coefficient evolution}

The Taylor Model is used in the present study to provide the input data of evolution of $F_{l}^{m n}$ at corresponding strains. Several other methods were developed to simulate the texture evolution based on different assumptions. Such methods include the self-consistent model (Lopes et al., 2003), the finite element analysis method (Nakamachi et al., 2000; Raabe and Roters, 2003; Kalidindi, 2001; Demirel et al., 2003; Houtte et al., 2002) and the Constrained Hybrid model (Parks and Ahzi, 1990; Ahzi et al., 1994). This work is neither to verify the correctness of the Taylor model, nor to develop a new model in terms of deformation mechanisms. Factors that will be considered in physical models, such as the constitutive relations in the slip systems, strain hardening and strain rate, are not considered at this stage. Prediction from Taylor model simulation and not experimental data are used since the Taylor model gives accurate estimates at different strains. The goal of our study is to propose an analytical form for the texture evolution and check its validity, limitations and applicability.

Using Eq. (11), the solution for $A_{\lambda l}^{\text {gpmn }}$ was obtained from known texture coefficients at different deformed states. To check the validity of this approach, texture coefficients $F_{l}^{m n}$ at other strains calculated from the resultant $A_{\lambda l}^{\sigma \rho m n}$ according to Eq. (12) are compared with those predictions from the Taylor model. For convenience, the initial texture in this study is arbitrarily assumed to be an aggregate of 400 crystals evenly distributed in the orientation space. The corresponding $(100)$, (1 110$)$ and (1 111$)$ pole figures of this data set are illustrated in Fig. 1. The maximum intensity is very small, less than 1.5 times random. $F_{0}^{11}, F_{4}^{11}, F_{4}^{12}$ and $F_{4}^{13}$ for this texture condition are $1.00,0.04,-0.05$, and 0.002 , respectively. All the other texture coefficients are zeros. The values are very close to the ideal random texture where only $F_{0}^{11}$ is 1 and all the other texture coefficients are zero.

For the present study, face centered cubic crystal system and orthotropic sample symmetry are assumed. In this system, the higher order texture coefficients $F_{l}^{m n}$ with $l>4$ (Eq. (2)) will not influence elastic properties (Bunge, 1982). As a result, a minimum value of 4 is chosen for the order of rank $l$ in Eq. (3). This greatly reduces the complexity of our problem. Furthermore, $F_{0}^{11}$ is a constant (always 1.0). This reduces the number of texture coefficients to only three terms: $F_{4}^{11}, F_{4}^{12}$ and $F_{4}^{13}$. That is to say, $N=3$. 
From Eq. (11), we have:

$$
\begin{aligned}
\frac{\mathrm{d} F_{4}^{11}(\eta)}{\mathrm{d} \eta} & =\sum_{\lambda=0}^{L_{\max }} \sum_{\sigma=0}^{N(\lambda)} \sum_{\rho=0}^{M(\lambda)} A_{l \lambda}^{m n \sigma \rho} F_{\lambda}^{\sigma \rho}(\eta) \\
& =A_{40}^{1111} F_{0}^{11}+A_{44}^{1111} F_{4}^{11}+A_{44}^{1112} F_{4}^{12}+A_{44}^{1113} F_{4}^{13}+A_{46}^{1111} F_{6}^{11}+A_{46}^{112} F_{6}^{12}+\cdots,
\end{aligned}
$$

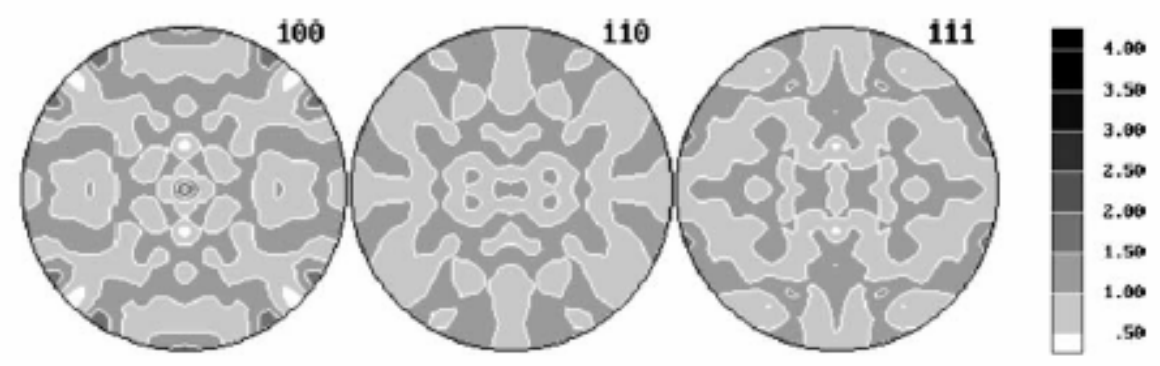

Fig. 1. (1 $\left.\begin{array}{lll}0 & 0\end{array}\right),\left(\begin{array}{lll}1 & 1 & 0\end{array}\right)$ and (1 111$)$ pole figures of initial simulated random texture by 400 crystals.

$$
\begin{aligned}
& \frac{\mathrm{d} F_{4}^{12}(\eta)}{\mathrm{d} \eta}=A_{40}^{1111} F_{0}^{11}+A_{44}^{1211} F_{4}^{11}+A_{44}^{1212} F_{4}^{12}+A_{44}^{1213} F_{4}^{13}+\cdots, \\
& \frac{\mathrm{d} F_{4}^{13}(\eta)}{\mathrm{d} \eta}=A_{40}^{1311} F_{0}^{11}+A_{44}^{1311} F_{4}^{11}+A_{44}^{1312} F_{4}^{12}+A_{44}^{1313} F_{4}^{13}+\cdots,
\end{aligned}
$$

or in matrix form,

$$
\left[\begin{array}{c}
\mathrm{d} F_{0}^{11} / \mathrm{d} \eta \\
\mathrm{d} F_{4}^{11} / \mathrm{d} \eta \\
\mathrm{d} F_{4}^{12} / \mathrm{d} \eta \\
\mathrm{d} F_{4}^{13} / \mathrm{d} \eta
\end{array}\right]=\left[\begin{array}{ccccc}
A_{00}^{1111} & A_{04}^{1111} & A_{04}^{1112} & A_{04}^{1113} & \ldots \\
A_{40}^{111} & A_{44}^{1111} & A_{44}^{1112} & A_{44}^{1113} & \ldots \\
A_{40}^{1211} & A_{44}^{1211} & A_{44}^{1212} & A_{44}^{1213} & \ldots \\
A_{40}^{1311} & A_{44}^{1311} & A_{44}^{1312} & A_{44}^{1313} & \ldots
\end{array}\right]\left[\begin{array}{c}
F_{0}^{11} \\
F_{4}^{11} \\
F_{4}^{12} \\
F_{4}^{13} \\
\vdots
\end{array}\right] .
$$

Here $A_{l \lambda}^{m n \sigma \rho}$ are components of a sixth order tensor reduced to a second rank tensor. Note that since $F_{0}^{11}$ is always equal to 1 , the rate of change of this coefficient is always 0 . It is clear that the summation over the right hand side does not need to be limited to $L_{\max }=4$ and actually depends on the character of the texture. Most of the components of this tensor are negligible because most of $F_{l}^{m n} \mathrm{~s}$ are either 0 or not of our interest. When $L_{\max }=4$, only 16 of the nonzero $A_{l \lambda}^{m n \sigma \rho}$ coefficients are shown in the matrix above. These coefficients will be used to describe the rate of change of text ure coefficients as a first estimate. The contribution from the higher order terms up to $L_{\max }=8$ will also be examined in the latter part of this work. The truncation error introduced by terminating at a finite value $\lambda=L_{\max }$ will be studied in a subsequent work. For simplification, only $L_{\max }=4$ is considered here for mathematical expansion, Eq. (14) is then rewritten in the following contracted form: 


$$
\left[\begin{array}{l}
\mathrm{d} F_{1} / \mathrm{d} \eta \\
\mathrm{d} F_{2} / \mathrm{d} \eta \\
\mathrm{d} F_{3} / \mathrm{d} \eta \\
\mathrm{d} F_{4} / \mathrm{d} \eta
\end{array}\right]=\left[\begin{array}{llll}
A_{10} & A_{11} & A_{12} & A_{13} \\
A_{20} & A_{21} & A_{22} & A_{23} \\
A_{30} & A_{31} & A_{32} & A_{33} \\
A_{40} & A_{41} & A_{42} & A_{43}
\end{array}\right]\left[\begin{array}{c}
F_{1} \\
F_{2} \\
F_{3} \\
F_{4}
\end{array}\right] .
$$

As described above, the sixth order tensor of text ure evolution coefficients is reduced to a second order matrix by filtering texture coefficients to only the ones which are of interest. After the calculation of the texture evolution matrix $A$, texture coefficients along the deformation history can be calculated by the texture evolution function:

$$
F(\eta)=\mathrm{e}^{A\left(\eta-\eta_{0}\right)} F\left(\eta_{0}\right) .
$$

In the present analysis, the texture coefficients at several different strains under uniaxial tension were obtained by the Taylor model. From these input data, texture evolution coefficients $A_{\lambda l}^{\sigma \rho m n}$ are calculated by solving Eq. (15). Next these coefficients will be used to simulate the evolution of texture coefficients at other strains in the deformation history according to Eq. (16). These simulated texture coefficients were compared with the Taylor prediction. Two methodologies are used to calculate the $A$ coefficients from the Taylor model. In the first attempt the data from the simulations are calculated for five different strains and the four simultaneous sets of equations are solved for the A coefficients. In the second method, texture data at a large number of strains are used and a least squares error method is used to calculate the A coefficients.

\subsection{Determined systems approach}

A number of points in the texture evolution paths are chosen and the data for the texture coefficients are calculated using the Taylor model. In the first set, a strain step $\mathrm{d} \eta$ of $5 \%$ was used. The $F_{l}^{m n}$, input data, at strains of $15 \%, 20 \%, 25 \%, 30 \%$ and $35 \%$ were calculated from Taylor model. Here these strains combined are called the initial strain set. From the rate of change of texture coefficient $\mathrm{d} F_{l}^{m n} / \mathrm{d} \eta$ and the texture coefficients $F_{l}^{m n}$ at different strains, $A_{\lambda l}^{\sigma \rho m n}$ were calculated. Using the texture evolution matrix $A$, the simulated values of texture coefficients $F_{l}^{m n}$ at strains from $0 \%$ to $50 \%$, were obtained. The evolution of these three texture coefficients with the strain is shown in Fig. 2. In the strains between $5 \%$ and $45 \%$, the recalculated $F_{4}^{11}$ from $A_{\lambda l}^{\sigma \rho m n}$ are very close to those obtained from Taylor model according to Fig. 2(a). When the strain is higher than $45 \%$, the recalculated $F_{4}^{11}$ begins to deviate from the Taylor prediction. The same trend is observed in the evolution curves of $F_{4}^{12}$ and $F_{4}^{13}$ from Fig. 2(b) and (c). In the range of initial strain set, from $5 \%$ to $40 \%$, the results from the linear model have a first order agreement with the Taylor prediction. In a typical case when the strain is $30 \%$, the difference between recalculated values and Taylor prediction is negligible. It deviates from the prediction of Taylor model when the strain is far from the initial strain set.

In the second set, a strain step of $2 \%$ was used. The initial strain set includes $22 \%$, $24 \%, 26 \%, 28 \%$ and $30 \%$. Fig. 2 shows that the texture evolution function using $A$ from this strain set works well in the strains from $20 \%$ to $40 \%$. If the strain is smaller than $20 \%, F_{4}^{11}$ and $F_{4}^{12}$ are under-predicted and $F_{4}^{13}$ is over-predicted. 
A similar procedure was applied with a strain step of $1 \%$. The initial strain set includes $23 \%, 24 \%, 25 \%, 26 \%$ and $27 \%$. Texture evolution matrix $A$ obtained from this procedure works well in the strain range close to the initial strain set. When the strain is larger than $30 \%$, the simulated texture coefficients evolution curve deviates from the raw data curve. At larger strains, the predictions for $F_{4}^{11}, F_{4}^{12}$ and $F_{4}^{13}$ are completely out of the acceptable range.

It is clear from the results above that the procedure introduced in this paper provides the best results when used for interpolation. This means that if the text ure coefficients are predicted at a strain which is within the range of initial strain set, the error is negligible. If this prediction is extended to strains out of the range of the initial strain set, the farther the strain is from the range of the initial strain set, the worse the prediction of the texture evolution. The choice of the initial strain set is critical in improving the accuracy of the modeling for the texture evolution.

In practice, the main application for this methodology is to calculate the A coefficients from the experimental data. One major problem for this application is that the experimental data for texture at different strains are usually not widely available. Furthermore, it may be necessary to predict the texture data outside of the range of

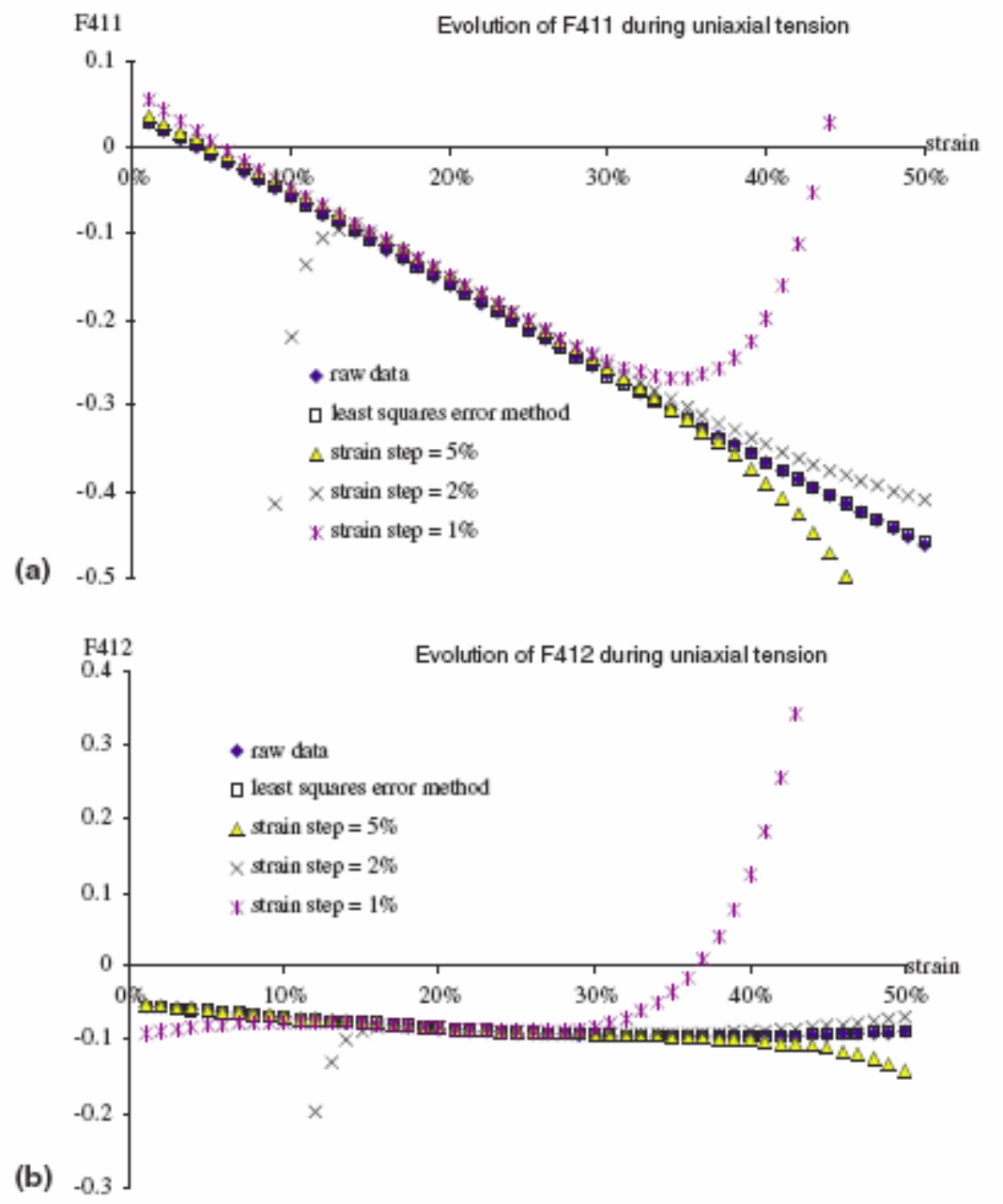




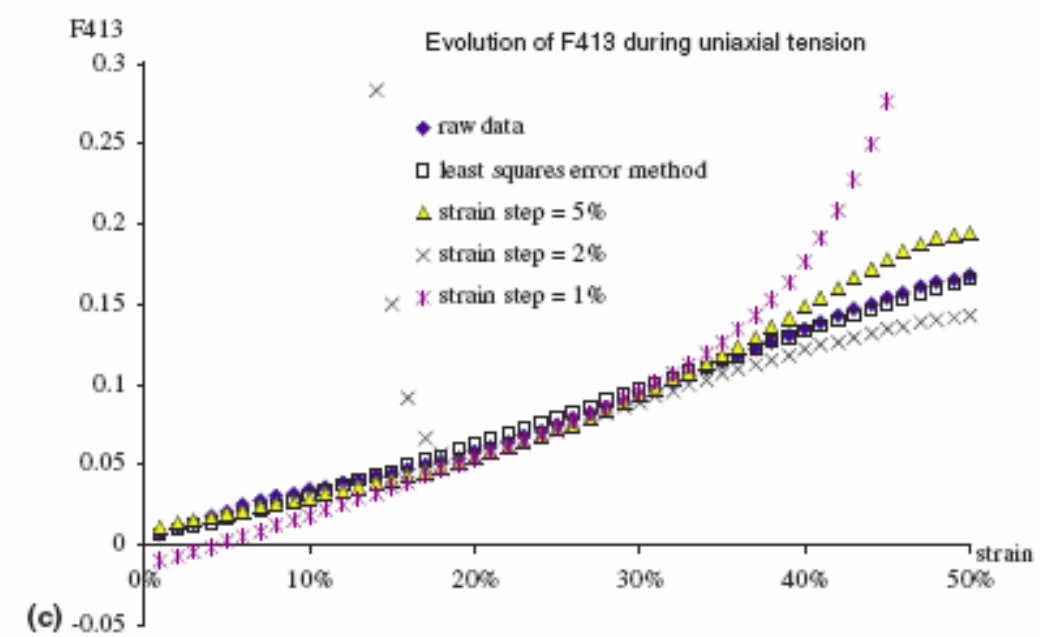

Fig. 2. Simulated processing path function during uniaxial tension of (a) $F_{4}^{11}$, (b) $F_{4}^{12}$ and (c) $F_{4}^{13}$ using texture evolution matrix $A$ obtained by strain step of $1 \%, 2 \%$ and $5 \%$, respectively. Evolution curves of $F_{4}^{11}, F_{4}^{12}$ and $F_{4}^{13}$ of Taylor prediction and simulation curve by least squares error method are also illustrated.

the available experimental data. Fully utilizing the strain range of the experimental data will increase the accuracy of this model to predict the texture evolution behavior outside of the experimental data.

\subsection{Least square errors method}

To improve the model's texture evolution predictions, the full range of the experimental data in the initial strain set is utilized. This was achieved by using the least squares error method to obtain the texture evolution coefficients.

If $M+1$ is the dimension of the initial strain set, there are $M$ simultaneous sets of equations. $N$ is the number of the rate of change of $F_{l}^{m n} \mathrm{~s}$ of interest. $P$ is then the number of $F_{\lambda}^{\sigma \rho}$ coefficients which are required to calculate each of the change rate of $F_{l}^{m n}$. From $M+1$ texture data $(M>N)$ at different strains, the texture evolution coefficient matrix $A$ was calculated from the over-determined system of equations as shown below:

$$
\left[\frac{\mathrm{d} F_{l}^{m n}(\eta)}{\mathrm{d} \eta}\right]=[A]\left[F_{\lambda}^{\sigma \rho}(\eta)\right] .
$$

$\mathrm{d} F_{l}^{m n}(\eta) / \mathrm{d} \eta$ is an $N \times M$ matrix. $A$ is an $N \times P$ matrix and $F_{\lambda}^{\sigma \rho}(\eta)$ is an $P \times M$ matrix.

Here the number of points $M$ is chosen as 10 ; the initial strain set varies from $30 \%$ to $41 \%$, and the strain step $\mathrm{d} \eta$ is $1 \%$. The range of this initial strain set is smaller than that used with strain step of $5 \%$, but larger than those used with the strain step of $2 \%$ and $1 \%$. From the texture data at these strains, $A_{\lambda l}^{\sigma \rho m n}$ coefficients were calculated by the least squares error method. The resultant $A_{\lambda l}^{\sigma \rho m n}$ s were used to recalculate the evolution of texture coefficients during the deformation from $20 \%$ to $50 \%$. To illustrate the advantage of the least squares error method, the results are also shown in Fig. 2. It can be seen that the least squares error method describes the behavior better than using a strain step of 5\%. The first result has a small initial strain set range (from $30 \%$ to $40 \%$ ) while the second result has a relatively large initial strain set range (from $25 \%$ to $45 \%$ ). The agreement with the Taylor prediction of the simulated results using least squares method is almost the same as using the strain step of $5 \%$ in the strain 
range of $25 \%$ to $45 \%$. This range is included in the initial strain set. When extrapolated outside of the initial strain range, the recalculated texture coefficients evolution curves using the least squares error method are closer to the curves from the Taylor prediction than any other simulated curves. The texture evolution coefficients obtained using least squares method give a more accurate description of the texture evolution behavior during mechanical deformation.

An error parameter, error ${ }_{l}^{m n}$, defined as the absolute value of the difference between the simulated texture coefficients and the Taylor prediction is introduced to demonstrate the accuracy of the procedure.

$$
\operatorname{error}_{l}^{m n}(\eta)=\operatorname{abs}\left(\left(F_{l}^{m n}\right)_{\text {simulated }}-\left(F_{l}^{m n}\right)_{\text {rawdata }}\right) .
$$

To compare the errors in a strain range, a mean error $\overrightarrow{\text { error }}_{1}^{\mathrm{mn}}$ parameter is defined as the average of the errors as calculated in Eq. (18) along the strain range:

$$
\overline{\text { error }}_{1}^{\mathrm{mn}}=\frac{1}{\mathrm{~N}} \sum \text { error }_{1}^{\mathrm{mn}}
$$

Fig. 3 illustrates the mean error parameter for the three nonzero texture coefficients obtained from different approaches. In the strain range from $20 \%$ to $50 \%$, the simulation from over-determined system using least squares error method fits best to the Taylor prediction. The mean errors of $F_{4}^{11}, F_{4}^{12}$ and $F_{4}^{13}$ from the over-determined system (least square errors method) are all smaller than the mean errors of the corresponding texture coefficients using the determined system method. The simulation from strain step of $5 \%$ is the next most accurate followed by the $2 \%$ strain step. For a strain step of $1 \%$, the mean error becomes very large. This large error occurs because the error is averaged in a strain range which is 10 times the range of its initial strain set. The analysis of the mean error corresponds to the analysis of simulation behavior of the previous texture evolution function.

\subsection{Influence of truncation limit for texture coefficients}

In all above simulations the maximum number of coefficients used in Eq. (3) was $L_{\max }=4$. Since texture representation requires a larger number of coefficients, there maybe a truncation error which may be reduced by increasing the number of coefficients. To check the influence of truncation error, the number the results for $L_{\max }=4$ was compared to $L_{\max }=8$ by increasing the number of coefficients to 8 . The initial strain set included $1 \%, 2 \%, 3 \%, \ldots, 50 \%$. The simulated results for the evolution of texture coefficient $F_{4}^{11}$ is presented in Fig. 4. The simulated evolution curve for $F_{4}^{11}$ from $L_{\max }=4$ (using the same initial strain set) is also shown here for 


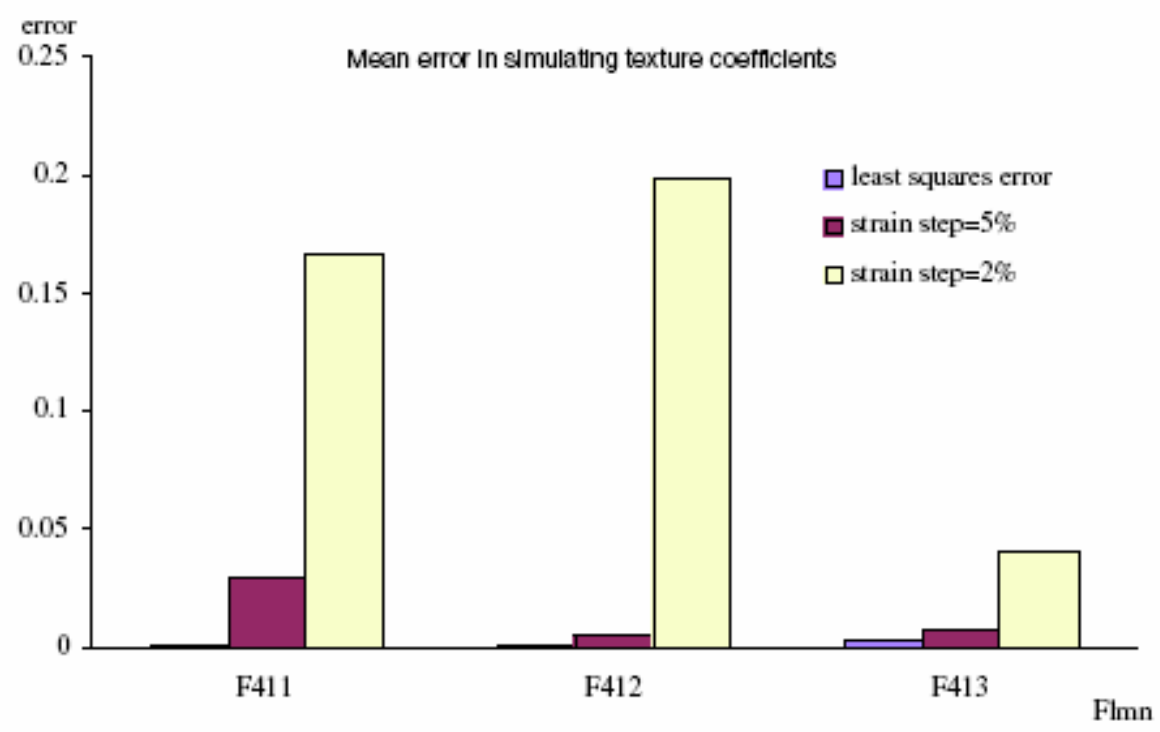

Fig. 3. error ${ }_{7}^{n}$, mean error of texture evolution of $F_{4}^{11}, F_{4}^{12}$ and $F_{4}^{13}$ during uniaxial tension when the simulated simulation results are obtained using different methods.

comparison. Using a higher truncation limit $(\operatorname{Lmax}=8)$, the predictions for the texture coefficients are closer to Taylor's results.

The texture coefficients for the initial microstructure has been selected near the origin of the texture hull, which represents a random state. The spherical harmonics method is efficient in representing the random or weak textured microstructures. This efficiency may have contributed to the small differences using the different truncation limits in Fig. 4. The results may be different if the initial microstructure is selected as a highly textured material. A full study of these other factor will be presented in a later work.

\subsection{Application of the texture evolution model for other processing paths}

This model has shown a remarkable success in representing texture evolution in uniaxial tension. It can also be applied to other processing paths including uniaxial compression test. From the same random texture, the evolution of the texture coefficients is illustrated in Fig. 5 for compression.

The Taylor prediction of $F_{4}^{11}, F_{4}^{12}$ and $F_{4}^{13}$ are shown in Fig. 5(a), (b) and (c), respectively. In a similar process, a strain step $\mathrm{d} \eta$ of $5 \%$ whose initial strain set includes $5 \%, 10 \%, 15 \%, 20 \%$ and $25 \%$ is used. Also, a least squares error method was used with the initial strain set of $1 \%, 2 \%, 3 \%, \ldots, 30 \%$. Fig. 5 shows that the resultant curves from these two simulation methods agree well with the curves from the predictions from the Taylor model. Least squares error method results in a small improvement in the prediction. In Fig. 6, mean error is used to compare the 


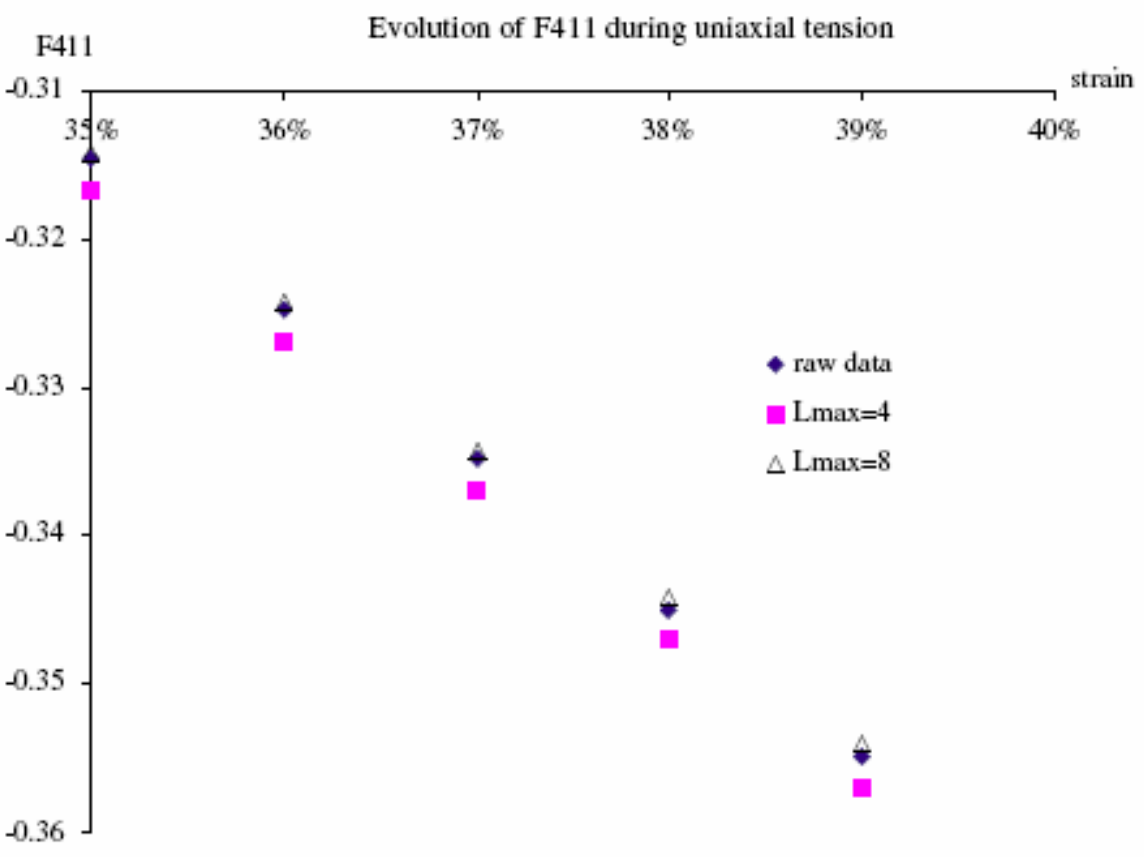

Fig. 4. Simulated processing path function of $F_{4}^{11}$ during uniaxial tension using texture evolution matrix $A$ obtained using $L_{\max }$ as 4 and 8, respectively.

(a)
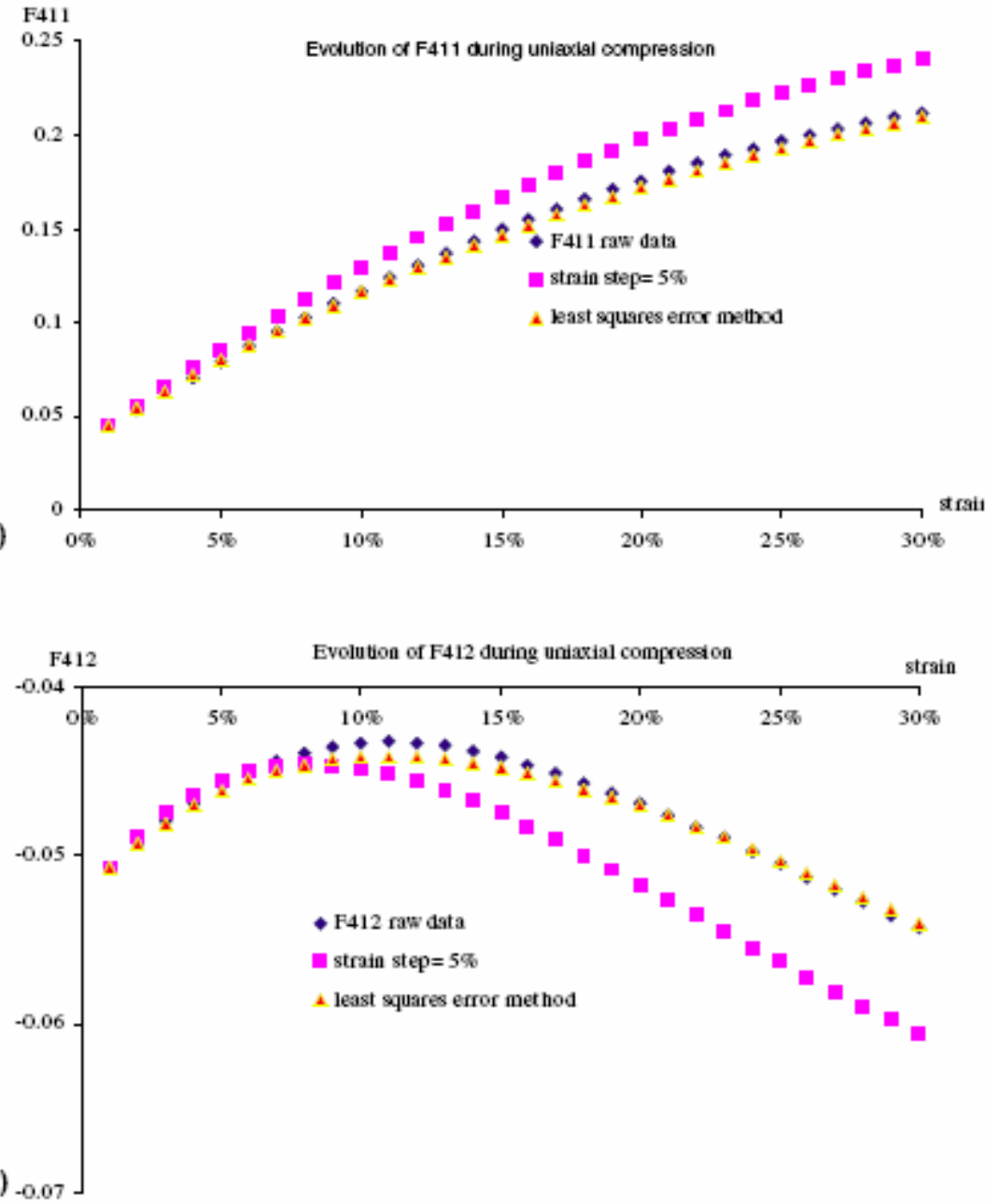


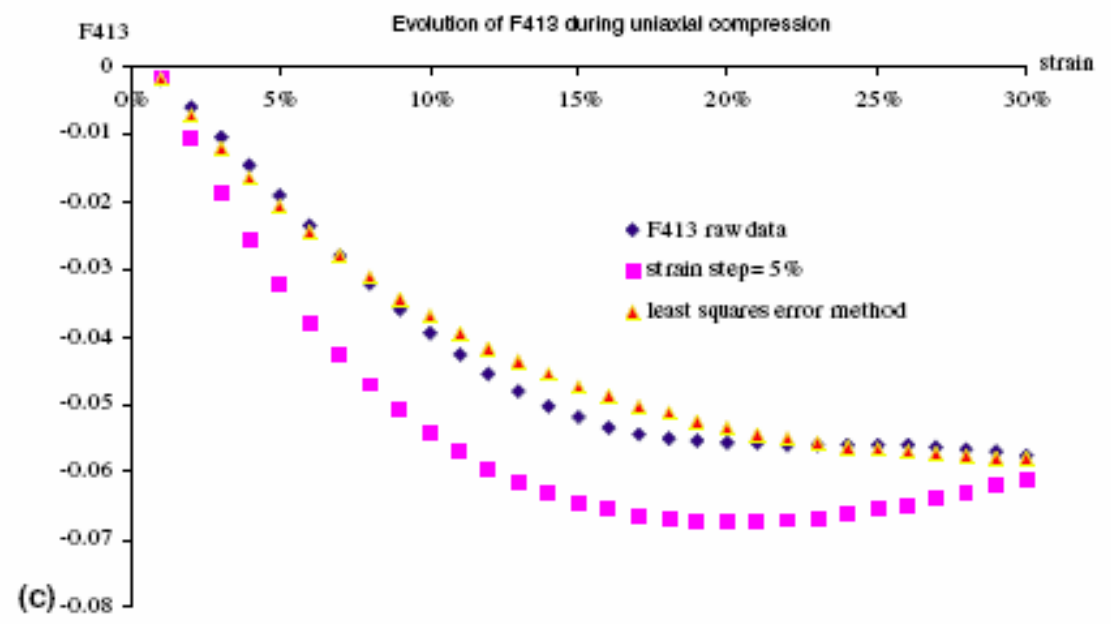

Fig. 5. Simulated processing path function during uniaxial compression of (a) $F_{4}^{11}$, (b) $F_{4}^{12}$ and (c) $F_{4}^{13}$ using texture evolution matrix $A$ obtained by strain step of $5 \%$, respectively. Evolution curves of $F_{4}^{11}, F_{4}^{12}$ and $F_{4}^{13}$ of Taylor prediction and simulation curve by least squares error method are also illustrated.

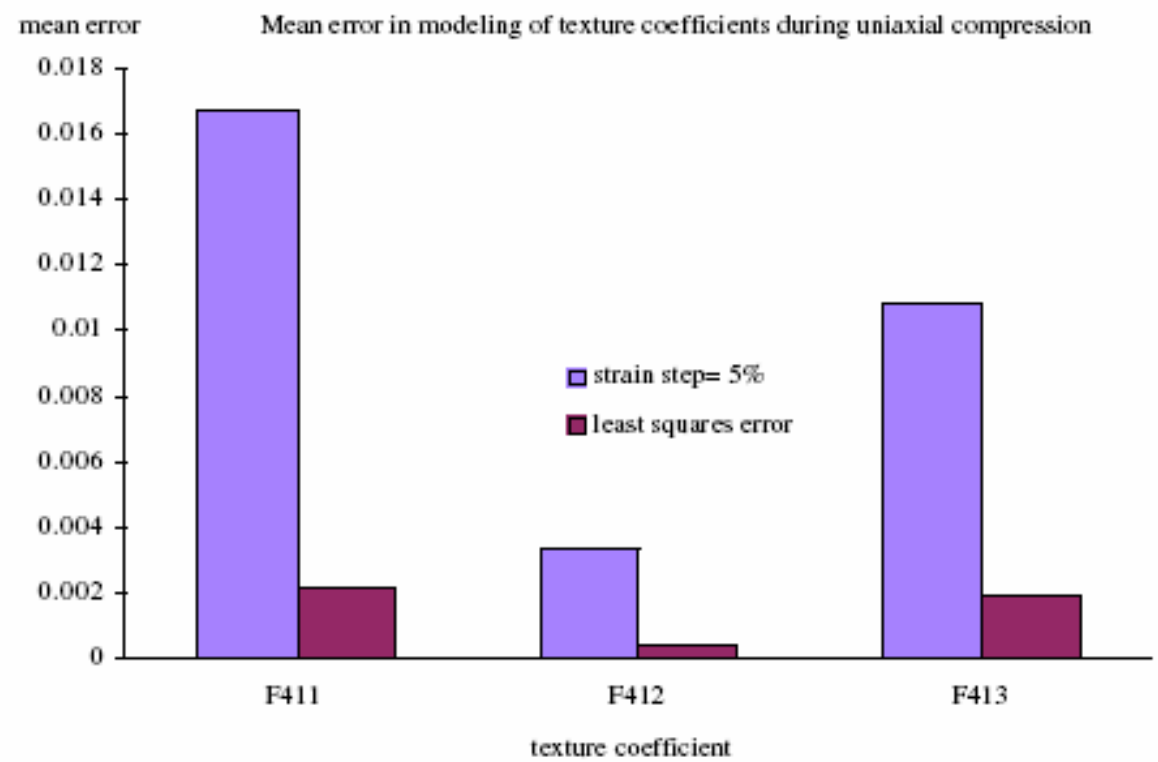

Fig. 6. error $_{l}^{\mathrm{N}}$, mean error of texture evolution of $F_{4}^{11}, F_{4}^{12}$ and $F_{4}^{13}$ during uniaxial compression when the simulated simulation results are obtained using different methods.

deviation from the Taylor prediction. In the strain range from $0 \%$ to $30 \%$, the mean error using least squares error method is only one-fifth to one-tenth of that using strain step $d \eta$ of $5 \%$.

Another state of stress used for the deformation process is rolling. From the same initial random texture, the evolution of the texture coefficients during rolling for a strain range between $0 \%$ and $30 \%$ is shown in Fig. 7 . The resultant simulated curves for the evolution of the texture coefficients are also illustrated in Fig. 7. The results show that the texture evolution model also fits the Taylor prediction in rolling and the simulation results from the least squares error method are in better agreement than that using strain step $\mathrm{d} \eta$ of $5 \%$. The mean errors of these two simulation methods for the strain range from $0 \%$ to $30 \%$ shown in Fig. 8 gives the same trend. 
Predictive range of the texture evolution model is not very impressive when it is applied to the Taylor simulation. It works well in the range close to the initial strain set. If the texture evolution matrix is applied to highly deformed microstructures or those quite different from the initial texture, the error is increased. This deviation may be attributed to the limitation of the Taylor model which is based on the assumption of homogeneous deformation rate throughout the material. This limitation leads to the limited predictive range of the conservation principle.

The limitation in using the simulation from Taylor is also revealed in the restriction of the predictive range of the texture evolution matrix $A$ for other points in the texture hull. The ideal texture evolution matrix $A$ should work for any microstructure with different textures as long as the same processing path is used. It is clear from this investigation that predictive range of $A$ is large but limited. For example, $A$ for uniaxial tension obtained from the initial strain set of $30 \%, 31 \%, \ldots, 40 \%$ using the least squares error method is applied to a randomly textured sample which was

(a)
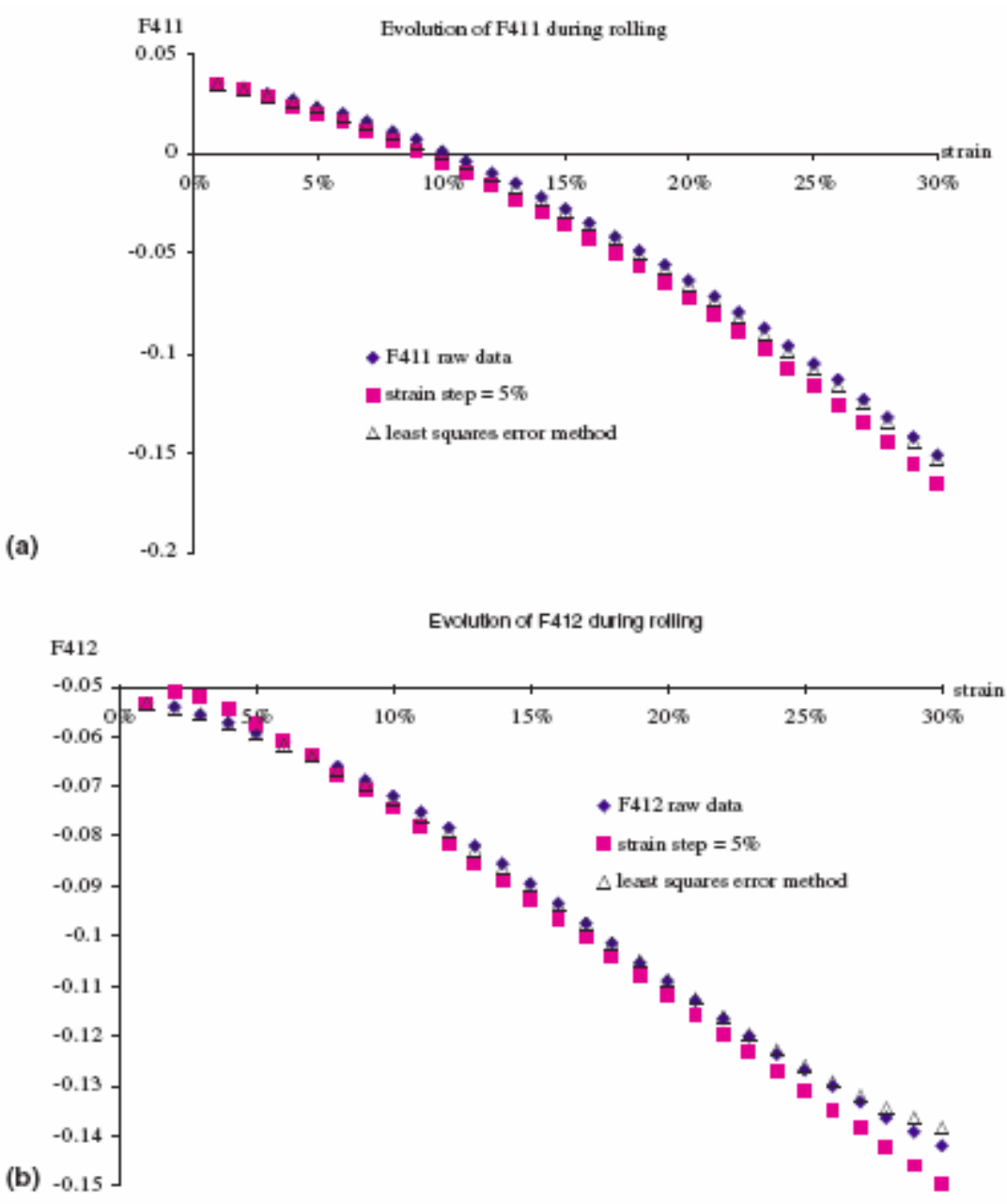

Evolution of F413 during rolling 


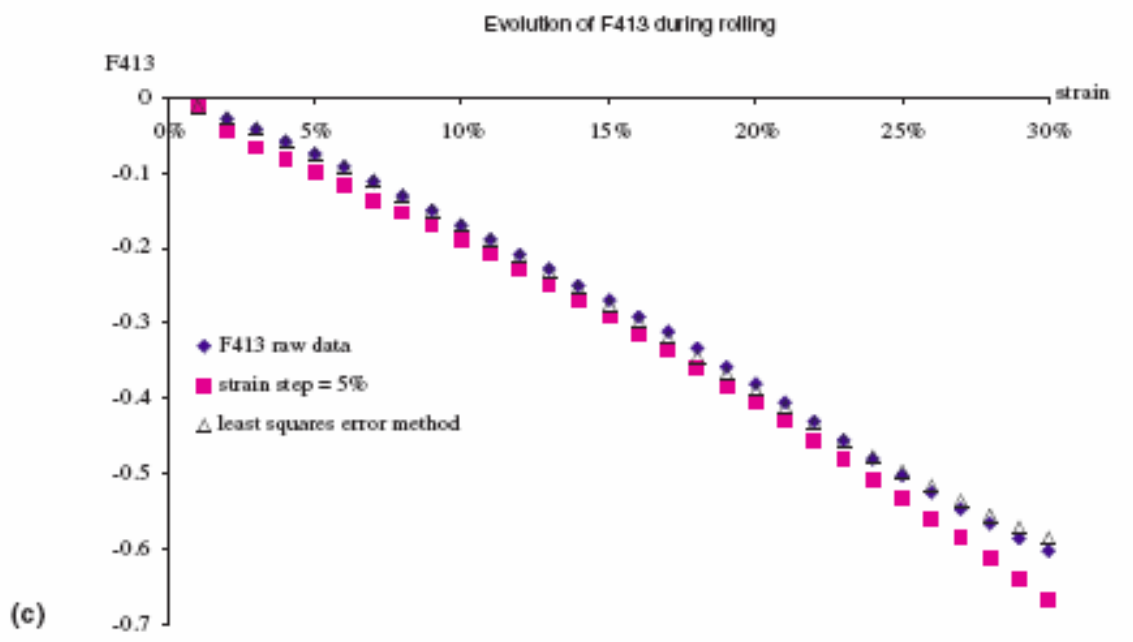

Fig. 7. Simulated processing path function during rolling of (a) $F_{4}^{11}$, (b) $F_{4}^{12}$ and (c) $F_{4}^{13}$ using texture evolution matrix $A$ obtained by strain step of $5 \%$, respectively. Evolution curves of $F_{4}^{11}, F_{4}^{12}$ and $F_{4}^{13}$ of Taylor prediction and simulation curve by least squares error method are also illustrated.

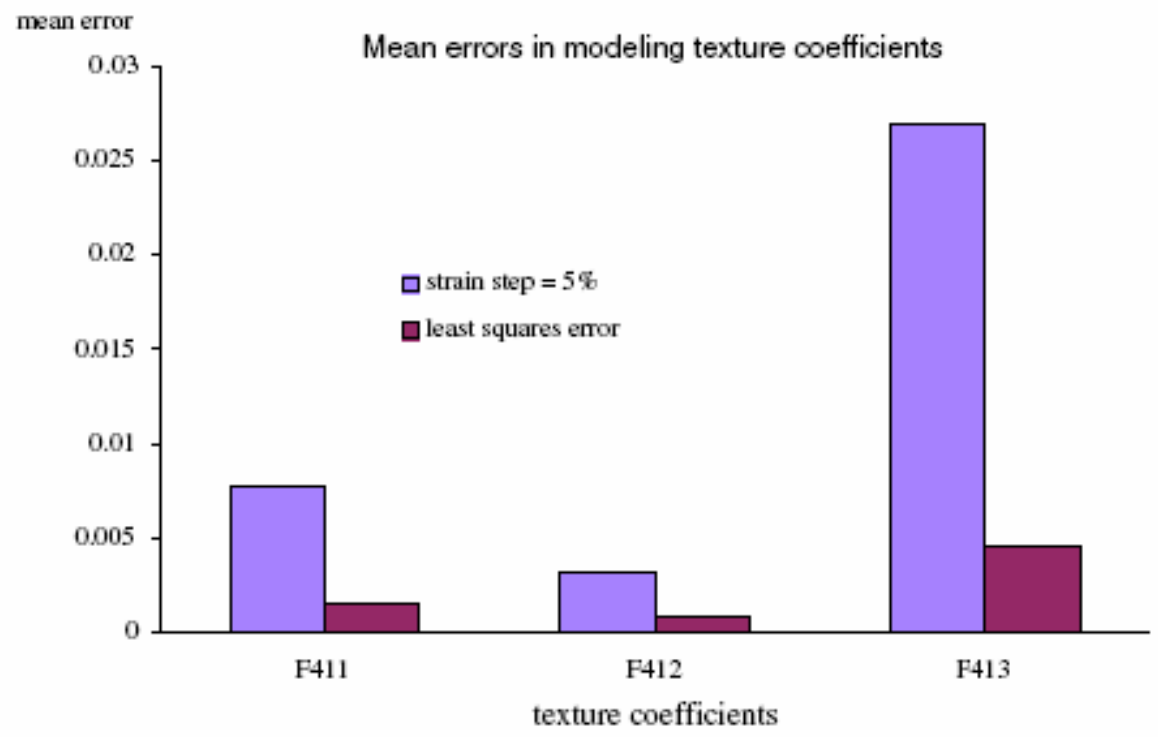

Fig. 8. $\overline{\operatorname{error}_{l}^{m}}$, mean error of texture evolution of $F_{4}^{11}, F_{4}^{12}$ and $F_{4}^{13}$ during rolling when the simulated simulation results are obtained using different methods.

rolled to 5\%. As shown in Fig. 9, the deviation of the present model for the texture evolution is very large when compared to Taylor's model. More work is needed to improve the predictive capability of the texture evolution matrix. Increasing the truncation limit for the texture coefficients may improve the error as mentioned in the earlier section. 


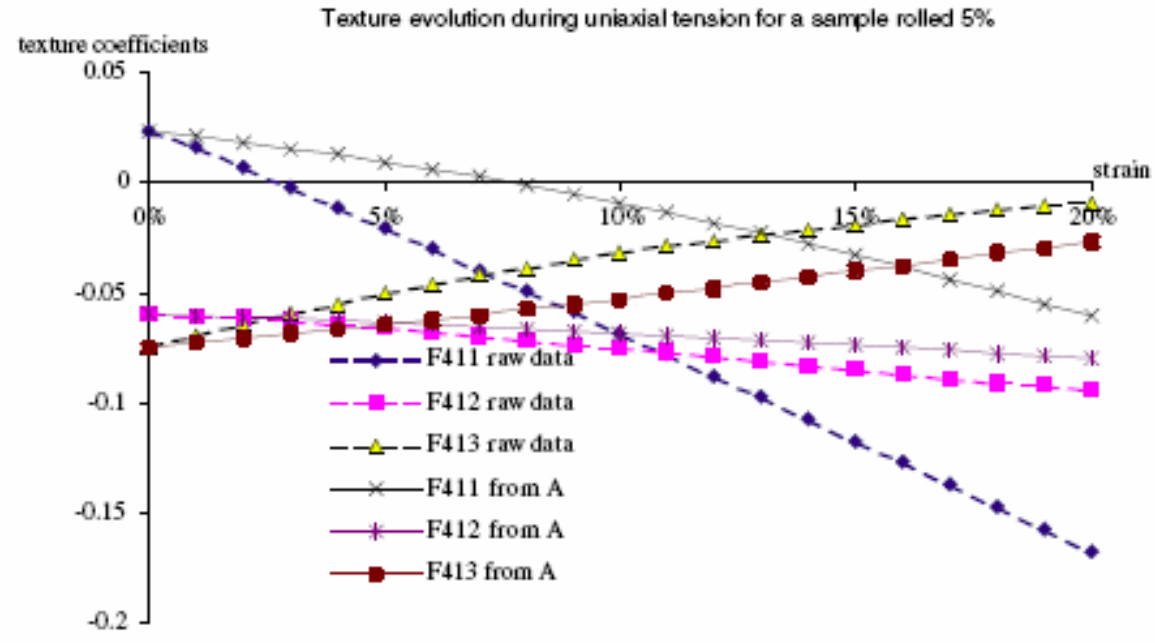

Fig. 9. Solid curves illustrate Taylor prediction of $F_{4}^{11}, F_{4}^{12}$ and $F_{4}^{13}$ during uniaxial tension of sample rolled $5 \%$ first from random texture status obtained from Taylor model. Dashed curves illustrate simulation result of $F_{4}^{11}, F_{4}^{12}$ and $F_{4}^{13}$ from $A$ calculated from the initial strain set of $30 \%, 31 \%, \ldots, 40 \%$ (uniaxial tension strain start from a random status) using the least squares error method.

\section{Texture evolution path in texture hull}

As described before, for the cubic-orthotropic system, three nonzero texture coefficients, $F_{4}^{11}, F_{4}^{12}$ and $F_{4}^{13}$ are sufficient to describe the texture in studying elastic properties. In a 3D space whose Cartesian coordinate axes are composed of these three texture coefficients, any point represents a microstructure (with a unique texture) that is related to its corresponding properties. The microstructure at any deformation state can then be represented by a point with coordinates represented by the texture coefficients. Texture evolution path is then a curve connecting the points during a single deformation process. Fig. 10 shows the texture evolution paths of 


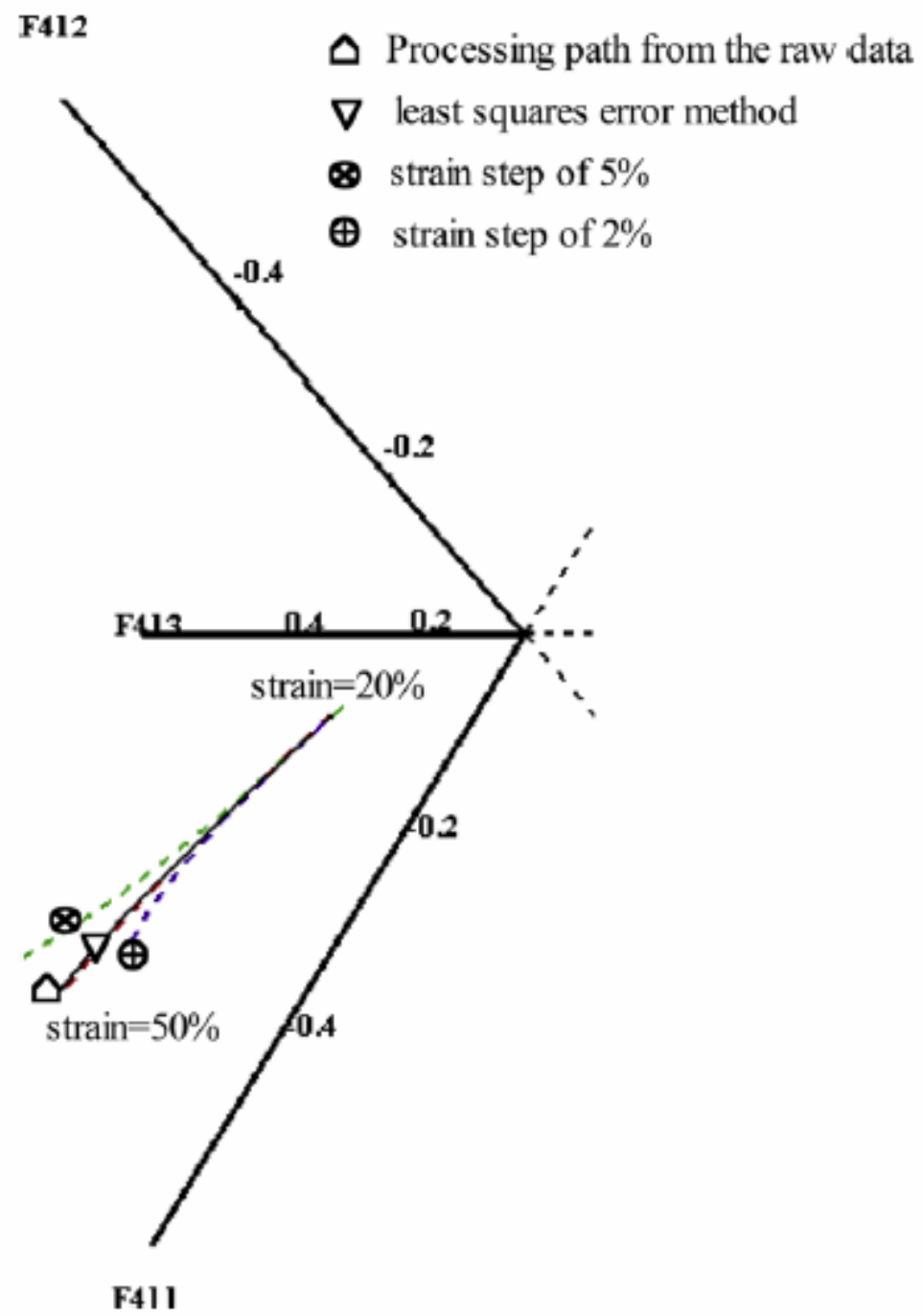

Fig. 10. Processing path from the Taylor prediction and simulated result presented in the texture hull whose coordinates are three texture coefficients $F_{4}^{11}, F_{4}^{12}$ and $F_{4}^{13}$. Solid line is Taylor prediction. Dash line denoted by symbol $\nabla$, very close to the black line, is from simulated result using least squares error method. Dash line for the simulation from strain step of $5 \%$ and $2 \%$ are denoted by symbol $\otimes$ and $\oplus$ respectively.

cubic-orthotropic system from Taylor's simulation and the texture evolution path model. The results describe the texture evolution from a strain of $20 \%$ to a strain of $50 \%$ during uniaxial tension. Using the model proposed in this paper, the evolution of texture coefficients as a function of the deformation is obtained. This model describes a simple but effective methodology to connect the evolution of microstructure and processing. The solid texture evolution path from the Taylor prediction is indistinguishable from the red dashed texture evolution path lines simulated using least squares error method. The dashed texture evolution path line denoted by symbol $\otimes$ represents the simulation result by a strain step of $5 \%$. It deviates a little from the texture evolution path as constructed from the Taylor prediction. The texture evolution path lines simulated by a strain step of $2 \%$ shows a larger deviation. The application of texture evolution path in MSD becomes very convenient and more understandable when it is constructed in the texture hull. Illustrated in Fig. 11, the texture hull is a compact convex subspace. All textures (representing microstructures) can only exist inside this convex subspace such that no point can exist outside 
of the wire-framed texture hull. The texture evolution path in Fig. 11 shows the simulated texture evolution from a textured state $\left(F_{4}^{11}=1, F_{4}^{12}=0, F_{4}^{13}=0\right)$ to a strain

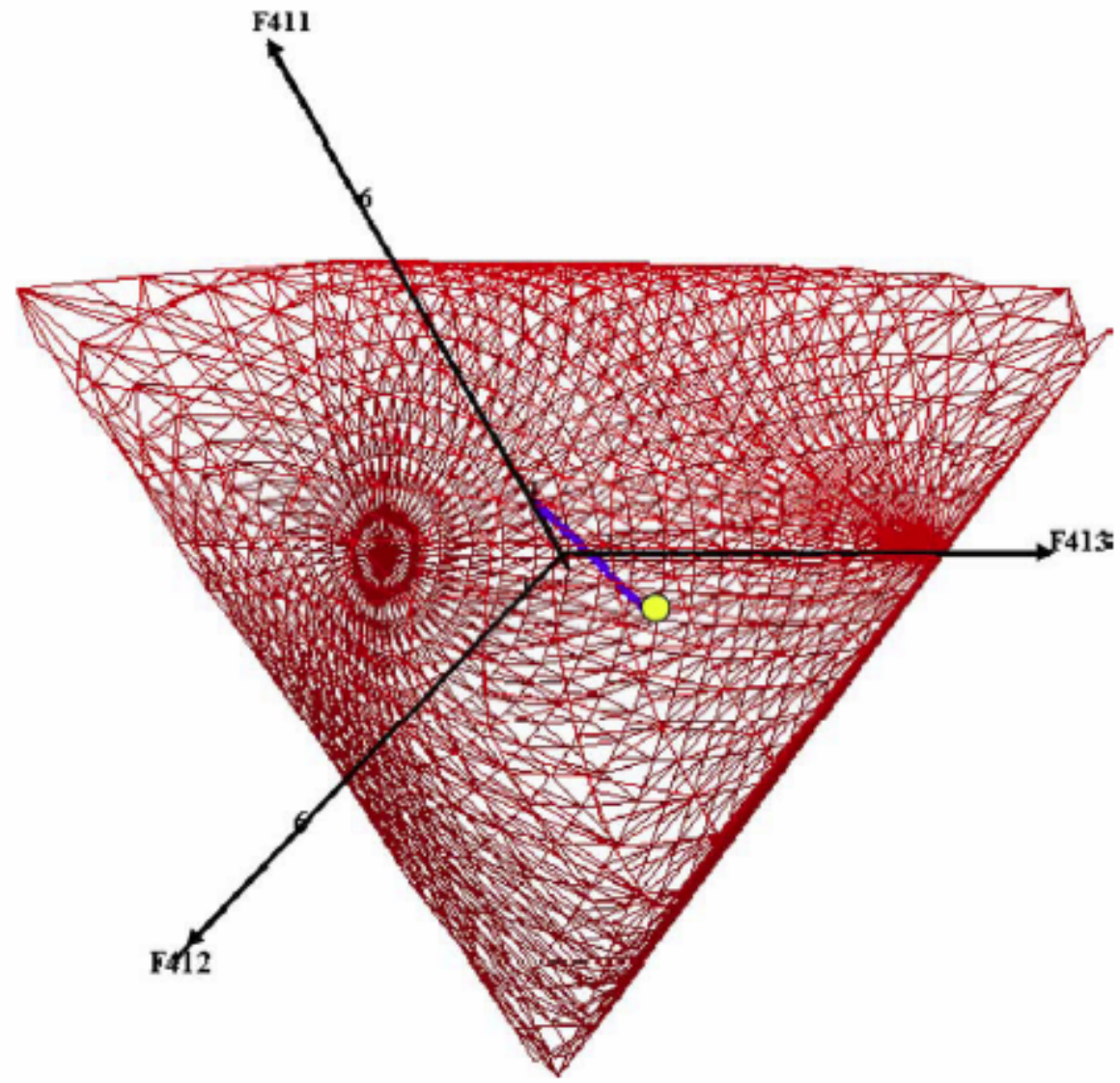

Fig. 11. Processing path of uniaxial tension from an initial state $\left\{F_{4}^{11}, F_{4}^{12}, F_{4}^{13}\right\}=\{1,0,0\}$ to a strain of $50 \%$ from the simulated result using least squares error method in the wire-framed texture hull. The staring point at a strain of $0 \%$ is close to original point. Yellow circle labels the microstructure at a strain of $50 \%$.

of $50 \%$ during uniaxial tension. The texture evolution matrix was obtained from the least squares error method for this simulation. The starting point is very close to origin of the coordinate system because the first three texture coefficients of the original random state are all zero. Using MSD, properties closure (Adams et al., 2001, 2004; Kalidindi et al., 2004) can be obtained by the intersection of several hyperplanes and may result in a subspace in the texture hull. Similarly, properties along the texture evolution path can be obtained by simple arithmetic averages of these microstructure components using Taylor's model as upper bound and Sach's model as lower bound. Taylor's model assumes a uniform deformation gradient for all grains in the polycrystalline materials and identical to the macroscopic deformation gradient. Sachs model assumes uniform stress throughout all the grains. Constrained-hybrid model adds the assumption of zero extension along some specific crystal directions (Parks and Ahzi, 1990). These models all require a clear understanding of the underlying deformation mechanism. They are applied in different materials and different deformation stages. The model proposed here in this study can simulate the texture evolution from the experimental measurement of textures without fully understanding the underlying deformation mechanisms. The model can clearly work best for interpolation as long as the texture evolution matrix $(A)$ can be determined from the experimental data. Extrapolation seems to give large deviation from 
the expected results when textures are predicted farther away from the initial strain set. Improving the deformation mechanisms may add some constraints and improve the predictive capability.

In this work only uniaxial tension, compression and rolling were studied. The same methodology can be implemented in obtaining texture evolution functions for other processing methods, such as biaxial tension, processing in magnetic field and so on. With the broadened knowledge, the processing path from one initial microstructure to a desired microstructure can be achieved by a combination of these texture evolution path functions.

\section{Streamlines for the evolution of texture coefficients}

The texture evolution function, as described earlier, is a function of the processing parameter $h$. This may impose a restriction on the use of the present model to a variety of optimization processes. In the spirit of Microstructure Sensitive Design, it is desirable to get the family of all texture evolution path functions for a specific processing path. This means that independent of the initial texture (microstructure), a materials designer may wish to explore all the different texture evolution paths to examine the family of microstructures that may be achieved by a single process (rolling, etc.). In this section, the streamline functions will be derived from the texture evolution functions. If the evolution of texture coefficients for polycrystalline materials in the texture hull is considered as a fluid flow, streamlines can then describe the texture evolution independent of the path parameter, $h$. In the following, the streamline for the evolution of the texture coefficients will be derived. Suppose the eigenvalues of $A$ are $a_{1}, a_{2}, \ldots, a_{n}$ and the corresponding eigenvectors are: $\vec{a}_{1}, \vec{a}_{2}, \ldots, \vec{a}_{n}$.

$$
\text { Let } L=\left[\begin{array}{llll}
a_{1} & & & \\
& a_{2} & & \\
& & \ddots & \\
& & & a_{n}
\end{array}\right]
$$

and

$$
P=\left[\begin{array}{llll}
\vec{a}_{1} & \vec{a}_{2} & \cdots & \vec{a}_{n}
\end{array}\right]
$$

Now it is clear that the evolution matrix $A$, (or the sixth rank tensor) can be decomposed as:

$$
A=P L P^{-1}
$$

Substituting this equation back into the texture evolution path function (12), we obtain:

$$
F(\eta)=\mathrm{e}^{P L P^{-1} \eta} F\left(\eta_{0}=0\right) .
$$


Further use of Eqs. (20) and (21), we have:

$$
F(\eta)=P \mathrm{e}^{L \eta_{\eta}} P^{-1} F\left(\eta_{0}=0\right)=P\left[\begin{array}{llll}
\mathrm{e}^{a_{1} \eta} & & & \\
& \mathrm{e}^{a_{2} \eta} & & \\
& & \ddots & \\
& & & \mathrm{e}^{a_{n} \eta}
\end{array}\right] P^{-1} F\left(\eta_{0}=0\right) .
$$

The same operation is executed on both sides of Eq. (24) to get:

$$
P^{-1} F(\eta) \cdot \mathrm{e}^{m}=\left[\begin{array}{llll}
\mathrm{e}^{a_{1} \eta} & & & \\
& \mathrm{e}^{a_{2} \eta} & & \\
& & \ddots & \\
& & & \mathrm{e}^{a_{n} \eta}
\end{array}\right] P^{-1} F\left(\eta_{0}=0\right) \cdot \mathrm{e}^{m} .
$$

$\mathrm{e}^{m}$ is a unit vector whose elements are 0 except the $m$ th element.

That is:

$$
\frac{P^{-1} F(\eta) \cdot \mathrm{e}^{m}}{P^{-1} F\left(\eta_{0}=0\right) \cdot \mathrm{e}^{m}}=\mathrm{e}^{a_{m} \eta}
$$

From here the texture evolution path parameter is obtained:

$$
\eta=\frac{\ln \left(\frac{P^{-1} F(\eta) \cdot e^{m}}{P^{-1} F\left(\eta_{0}=0\right) \cdot e^{m}}\right)}{a_{m}} .
$$

Substituting the evolution path parameter back to the texture evolution path function, we get the expression of the streamline of the texture coefficients:

$$
F(\eta)=\exp \left(\frac{\ln \left(\frac{P^{-1} F(\eta) e^{m}}{P^{-1} F\left(\eta_{0}\right) \cdot e^{m}}\right)}{a_{m}} A\right) F\left(\eta_{0}=0\right) .
$$

Fig. 12 shows the streamlines for texture evolution in $\left\{F_{l}^{\mu \nu}\right\}$ space during uniaxial tension. The only variable is the initial texture. The five streamlines shown here are from five different microstructures, whose $\left\{F_{4}^{11}, F_{4}^{12}, F_{4}^{13}\right\}$ are $\{0.2,0,0\},\{0.4,0,0\},\{0.6,0,0\},\{0.8,0,0\}$ and $\{1.0,0,0\}$, respectively. The same texture evolution matrix $A$ calculated by the least squares error method in Section 3.2 is used for the calculation of these five streamlines. The texture evolution can now be compared to the steady-state condition. There is no change in the direction of the velocity vector at any point. This means that the streamline is unique if the initial texture and processing method are the same.

The continuity relations in the Euler space can reduce a large set of constitutive relations and all its details into a sixth order tensor " $A$ " for relatively large strains. Such a reduction and its consequent set of streamlines can make the optimization for the texture evolution path real simple and save the designer a large amount of time. The 
following example is provided to explain the main utility of the texture evolution path parameter.

Texture can be represented by a set of texture coefficients as in Eq. (3). The first two nonzero texture coefficients are used in a two dimensional plot as in Fig. 13(a).

An off-the-shelf material (manufactured by casting and rolling) maybe represented by point $A$ in this two-dimensional plot. The material represented by point $A$, however, may not be the desired material and we may want to process a desired microstructure represented by point ' $B$ '. The question that may be raised is how the material represented by $A$ can be processed to become a material represented by $B$. Based on the present plasticity formulations we may have to depend on a large data base and the correct answer may lie in a large number of crystal plasticity set of simulations. The present paper provides a time saving and robust methodology to get to the material $B$ with texture represented by Eq. (3) by selecting one or a multiple number of paths. If the family of all possible deformations paths are considered for both $A$ and $B$ (as in Fig. 13(a)) the solution may be a combination of texture evolution paths connecting the two points (Fig. 13(b)). The solution is obviously not unique but using the streamlines formulation developed in the present paper we can choose a path which can best fit the designers objective. 


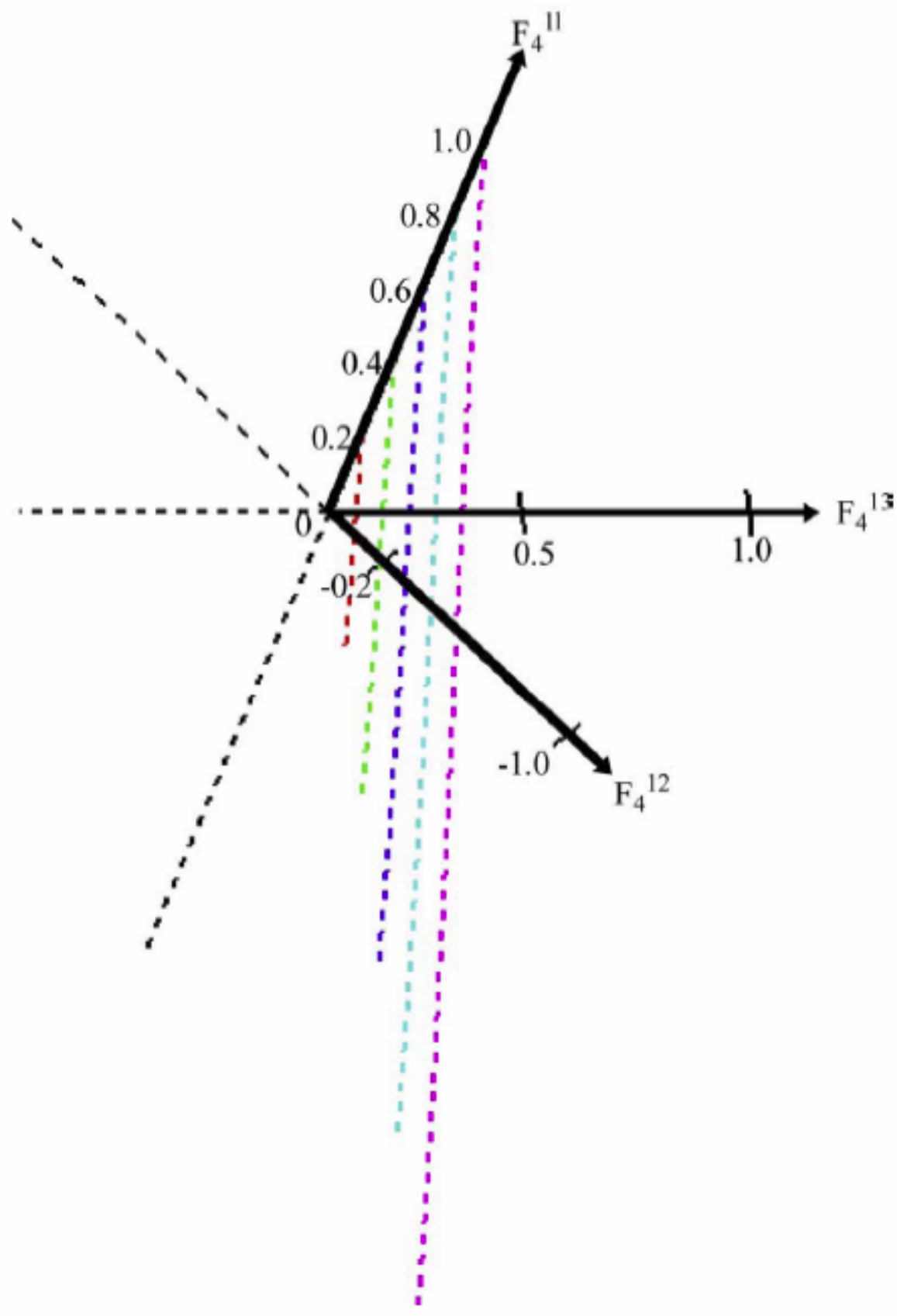

Fig. 12. Streamline of uniaxial tension calculated from the texture evolution matrix obtained from Taylor's model.

Texture is considered a macroscopic manifestation of the microstructure crystal grain orientation through the continuity relations in the Euler space. The results show that the continuity relations work in a large strain range which is a very important factor proving the accuracy of this principle. Remember that we use the continuity relations in the materials coordinate as part of the field equations in many of the formulations without 


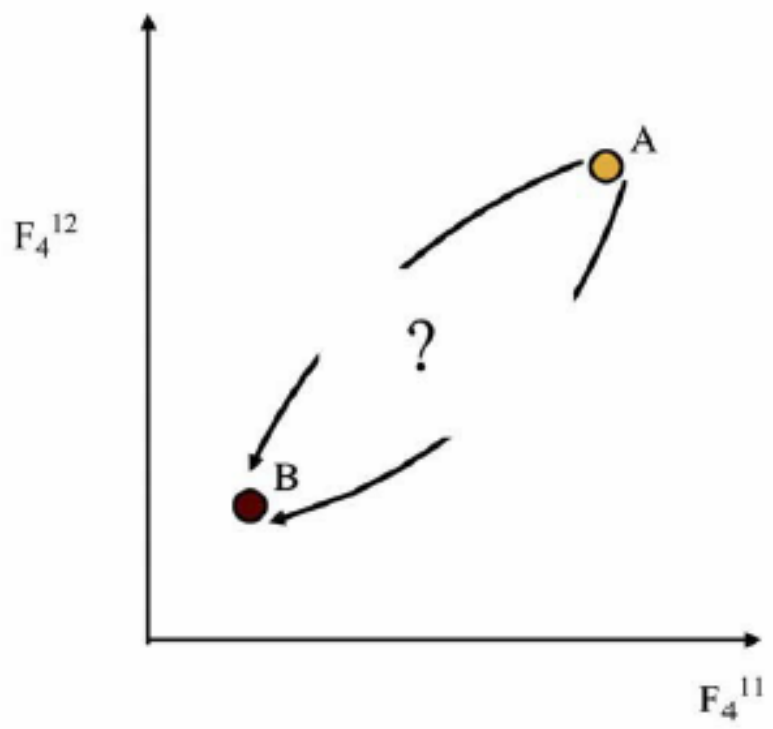

(a)

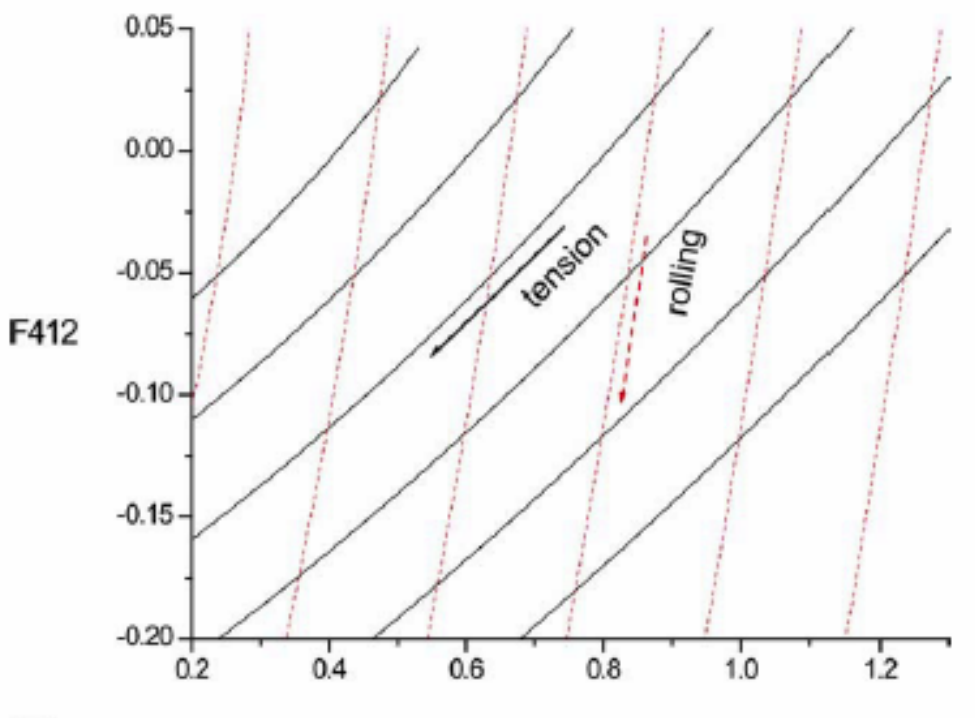

(b)

F411

Fig. 13. (a) Materials design problem presented in a simplified two dimensional materials space. (b) Streamline grid in the materials spaces present the solution for materials design.

regards to the underlying mechanisms for the deformation processes. It is clear that the continuity relations in the materials coordinate may also reach its limits and can only apply when the material can be assumed a continuous medium throughout the deformation process. The continuity relations in the orientation space can also reach its limits of usefulness when there is a discontinuity in the orientation space. This will occur during recrystallization and may occur in a number of other situations. 


\section{Conclusion and perspectives}

A conservation principle for texture evolution has been utilized to get a texture evolution path function. The solution also provides streamlines during any mechanical deformation process (rolling forging, drawing, etc.). Using texture coefficients as a descriptor, microstructures can be represented as points in the multidimensional space whose axes are these texture coefficients. Texture evolution path, a trace of texture coefficients during deformation history, is used to represent the microstructure evolution in this multidimensional space.

The texture evolution matrix $A$ is a critical parameter in the processing path function developed here. It is calculated from texture coefficients at different levels of deformation defined as the initial strain set. The simulation works well as a predictive tool if the desired microstructure (texture) is calculated for deformations in the range of the initial strain set. Increasing the range of the initial strain set will improve the performance of simulated result in a larger strain range. If more data are available to calculate the texture evolution matrix, utilization of the least squares error method will increase the accuracy of simulated texture evolution path.

Presenting the results of the texture evolution path in the texture hull shows how the microstructure evolves with the deformation parameter. Streamlines obtained from the texture evolution path functions give a representation of the texture evolution independent of the deformation parameter. Streamlines can simplify the optimization of the processing path and provide the mechanical designers with an important tool in Microstructure Sensitive Design.

\section{Acknowledgements}

This work has been funded under the AFOSR Grant \# F49620-03-1-0011 and Army Research Lab Contract \# DAAD17-02-P-0398 and DAAD 19-01-1-0742. "B.L. Adams acknowledges support of Army Research Office, Proposal No. 42566-MS.' 'The authors express their sincere gratitude to Professor Surya Kalidindi, for helpful suggestions and correspondence for the derivation of the processing path functions.

\section{References}

Adams, B.L., Canova, G.R., Molinari, A., 1989. A statistical formulation of viscoplastic behavior in heterogeneous polycrystals. Textures Microstruct. 11, 57-71.

Adams, B.L., Henrie, A., Henrie, B., Lyon, M., Kalidindi, S.R., Garmestani, H., 2001. Microstructuresensitive design of a compliant beam. J. Mech. Phys. Solids 49, 16391663.

Adams, B.L., Lyon, M., Henrie, B., 2004. Microstructure by design: linear problems in elastic-plastic design. Int. J. Plast. 20, 1577-1602.

Ahzi, S., Lee, B.J., Asaro, R.J., 1994. Plasticity and anisotropy evolution in crystalline polymers. Mater. Sci. Eng. 189A, 35-44.

Bunge, H.J., 1965. Zur darstellung allgemeiner texturen. Z. Metallkde 56, 872-874. 
Bunge, H.J., 1982. Texture Analysis in Materials Science: Mathematical Methods. Butterworth, London, p. 340.

Bunge, H.J., Esling, C., 1984. Texture development by plastic deformation. Scripta Met. $18,191-195$.

Klein, H., Bunge, H.J., 1991. Modeling deformation texture formation by orientation flow-fields. Steel Res. 62, 548-559.

Clement, A., Coulomb, P., 1979. Eulerian simulation of deformation textures. Scripta Met. 13, 899-901.

Clement, A., 1982. Prediction of deformation texture using a physical principle of conservation. Mater. Sci. Eng. 55, 203-210.

Demirel, M.C., Kuprat, A.P., George, D.C., Rollett, A.D., 2003. Bridging simulations and experiments in microstructure evolution. Phys. Rev. Let. 90, Art. No. 016106.

Garmestani, H., Kalidindi, S.R., Williams, L., Bacaltchuka, C.M., Fountain, C., Lee, E.M., Se-Said, O.S., 2002. Modeling the evolution of anisotropy in Al-Li alloys: application to Al-Li 2090-T8E41. Int. J. Plast. 18, 1373-1393.

Garmestani, H., Harris, K., 1999. Orientation determination by EBSP in an environmental scanning electron microscope. Scripta Mater. 41, 47-53.

Garmestani, H., Kalu, P., Dingley, D., 1998. Micro-characterization of Al-8090 superplastic materials using orientation imaging microscopy. J. Mater. Sci. Eng. A 242, 284-291.

Garmestani, H., Lin, S., Adams, B., Ahzi, S., 2001. statistical continuum theory for texture evolution of polycrystals. J. Mech. Phy. Solids 49, 589-607.

Garmestani, H., Lin, S., 2000. Statistical continuum mechanics analysis of an elastic twoisotropic-phase composite material. J. Compos. B 31, 39-46.

Houtte, P.V., Delannay, L., Kalidindi, S.R., 2002. Comparison of two grain interaction models for polycrystal plasticity and deformation texture prediction. Int. J. Plast. 18, 359-377.

Jefferson, Garmestani, H., Tannenbaum, R., Gokhale, A., Tadd, E.. Two-point probability distribution function analysis of co-polymer nano-composites. Int. J. Plast. 21, $185-198$.

Kalidindi, S.R., Bronkhorst, C.A., Anand, L., 1992a. Crystallographic texture evolution in bulk deformation processing of fcc metals. J. Mech. Phys. Solids 40, 537-569.

Kalidindi, S.R., Anand, L., 1992b. An approximate procedure for predicting the evolution of crystallographic texture in bulk deformation processing of fcc metals. Int. J. Mech. Sci. 34, 309-329. 
Kalidindi, S.R., 2001. Modeling anisotropic strain hardening and deformation textures in low stacking fault energy fcc metals. Int. J. Plast. 17, 837-860.

Kalidindi, S.K., Houskampa, J.R., Lyons, M., Adams, B.L., 2004. Microstructure sensitive design of an orthotropic plate subjected to tensile load. Int. J. Plast. 20, 15611575.

Li, D.S., Garmestani, H., 2003a. Evolution of crystal orientation distribution coefficients during plastic deformation. Scripta Mater. 49, 867-872.

Li, D.S., Garmestani, H., 2003b. Dislocations, Plasticity and Metal Forming. NEAT Press, pp. 487-489.

Lin, S., Garmestani, H., Adams, B.L., 1998. Statistical continuum theory for inelastic behavior of twophase medium. Int. J. Plast. 14, 719-731.

Lin, S., Garmestani, H., Adams, B., 2000. The evolution of probability functions in an inelastically deforming two-phase medium. Int. J. Solids Struct. 37, 423-434.

Lopes, A.B., Barlat, F., Gracio, J.J., Duarte, J.F., Rauch, E.F., 2003. Effect of texture and microstructure on strain hardening anisotropy for aluminum deformed in uniaxial tension and simple shear. Int. J. Plast. 19, 1-22.

Nakamachi, E., Hiraiwa, K., Morimoto, H., Harimoto, M., 2000. Elastic/crystalline viscoplastic finite element analyses of single- and poly-crystal sheet deformations and their experimental verification. Int. J. Plast. 16, 1419-1441.

Parks, D.M., Ahzi, S., 1990. Polycrystalline plastic deformation and texture evolution for crystals lacking five independent slip systems. J. Mech. Phys. Solids 38, 701-724.

Raabe, D., Roters, F., 2003. Using texture components in crystal plasticity finite element simulations. Int. J. Plast. 20, 339-361.

Torquato, S., Stell, G., 1985. Microstructure of two-phase random media. V. The n-point matrix probability functions for impenetrable spheres. J. Chem. Phys. 82, 980-987. 